\title{
Aporte calórico y macronutrimental de los menús infantiles de la comida rápida y convencional
}

\author{
Caloric and macronutrient contribution of the children's \\ menu of fast and conventional food
}

\begin{abstract}
The caloric and macronutrient contribution of the children's menu in fast food restaurants and conventional restaurants was analyzed, according to mealtimes and recommended intakes for children between 4 and 8 years old in a city in the Northeastern of Mexico with the highest childhood obesity rate in the world. This is an observational, descriptive and transversal study. The sampling was by convenience and directed when typical cases of children's menus were selected from 152 restaurants. The range of carbohydrates contribution of the children's menu varied from $53.8 \%$ to $97.9 \%$ of the recommendation; in any case the protein contribution was less than $82.2 \%$. With regard to fats, contribution ranges were from $63 \%$ to $165.3 \%$. These contributions increase considerably when they are analyzed by mealtimes: from $97.8 \%$ to $373.4 \%$. In conclusion, fast food and conventional restaurants offer unhealthy meals in big portions to child population.

Key words: caloric and macronutrients contribution; conventional restaurants; fast food; children's menu; children nutrition.
\end{abstract}

\section{INTRODUCCIÓN}

Durante los últimos años en México comer fuera de casa ha sido un fenómeno creciente asociado al aumento de la oferta de todo tipo de alimentos. Una síntesis histórica de la Encuesta Nacional de Ingresos y Gastos de los Hogares (ENIGH) (1-5) permite observar que durante la última década el número de hogares que consumen alimentos y bebidas fuera del hogar tuvo un crecimiento de $63,7 \%$ y el gasto en este mismo rubro aumentó un 99,9\%.

Por otro lado, los Censos Económicos oficiales (6-12) llevados a cabo entre 1981 y 2009 por el Instituto Nacional de Estadística, Geografía e Informática (INEGI) registraron un aumento de más de $700 \%$ en la categoría "servicios de comida para llevar". También, para 2009 los restaurantes de "autoservicio, comida para llevar y otros restaurantes con servicio limitado" constituyen en conjunto alrededor de $97 \%$ frente a sólo menos de 3\% de los restaurantes con servicio completo (12).

A los restaurantes de autoservicio, comida para llevar y con servicio limitado se les conoce comúnmente como de "comida rápida" y se definen (13) como aquellos que preparan alimentos y bebidas para consumo inmediato donde el cliente ordena su pedido a través de un mostrador, barra, vía telefónica o auto y paga antes o al momento de la entrega, no cuentan con meseros y ofrecen un menú restringido basado principal-
Esteban Gilberto Ramos P.

Ana Elisa Castro-Sánchez

Adriana Zambrano M.

Georgina Mayela Núñez $R$. Sylvia Osorio de D.

Centro de Investigación en Nutrición y Salud Pública de la Facultad de Salud Pública y Nutrición. Universidad Autónoma de Nuevo León, México.

Dirigir la correspondencia a: Profesor

Esteban Gilberto Ramos P. Centro de Investigación en Nutrición y Salud Pública Facultad de Salud Pública y Nutrición Universidad Autónoma de Nuevo León, México E-mail:ana.castrosh@uanl.edu.mx

Este trabajo fue recibido el 26 de Octubre de 2011 y aceptado para ser publicado el 15 de Junio de 2012.

mente en pollo apanado frito, pizza, hamburguesas, papas fritas y bebidas gaseosas; es una comida de fábrica, altamente procesada, producida, congelada, distribuida y almacenada en cantidades industriales y servida en materiales desechables de un solo uso. Mientras que los restaurantes con servicio completo o "convencionales" ofrecen un menú amplio y platillos frescos elaborado por cocineros especializados, cuentan con personal que toma la orden, sirve los alimentos y bebidas y llevan la cuenta a la mesa (13).

Así pues, la población mexicana transforma sus hábitos alimentarios mostrando una creciente tendencia en el consumo de alimentos y bebidas fuera de casa, de autoservicio, congelados, precocinados y para llevar, los cuales son de fácil acceso, preparación o consumo y de bajo costo, sin embargo, como lo han mostrado diversos estudios (14-17) se trata por lo general de platillos, comidas o bocadillos de alta densidad energética, altos en grasas saturadas y trans, azúcar y sal, cuyo consumo habitual se ha asociado a la obesidad y a otras enfermedades crónicas como la diabetes tipo 2 (17-19).

Es importante considerar que si bien personas de todas las edades están expuestas al consumo de este tipo de comida, la publicidad se ha enfocado a atraer a la clientela infantil y a grado tal, que la industria alimentaria ejerce una especie de poder social sobre la población escolar (20-28). En este sentido, existen diversos estudios $(19,29-36)$ que han mostrado 
la relación entre obesidad infantil y consumo de alimentos hipercalóricos. En México entre 1999 y $2006(37,38)$ la prevalencia de sobrepeso y obesidad aumentó progresivamente entre los 5 y 11 años de edad (en niños pasó de 12,9\% a $21,2 \%$ y en niñas de $12,6 \%$ a $21,8 \%$ ) lo cual los ha colocado en el primer lugar mundial respecto a esta problemática. Sin embargo, poco se sabe respecto al estilo de vida y en particular sobre los hábitos y preferencias alimentarios de los menores; tampoco existen datos estadísticos lo suficientemente desagregados para conocer con mayor precisión qué y dónde comen los niños y las niñas. En este sentido un aspecto fundamental se refiere al desconocimiento sobre el contenido calórico y nutrimental de los menús que más frecuentemente se ofertan a la población infantil.

A pesar de que en México la obesidad infantil constituye un grave problema de salud pública (38) y que sus niños y niñas se encuentran posicionados en los primeros lugares mundiales en exceso de peso, existe muy poca información sobre el contenido macronutrimental de los menús infantiles; respecto a esto sólo se conocen algunas estimaciones que ciertas cadenas de restaurantes han elaborado y las cuales no consideran las necesidades nutricionales y recomendaciones acordes a la edad de los menores.

El presente trabajo forma parte de un proyecto de investigación más amplio referente a la transformación de hábitos alimentarios en el noreste de México. Se aborda específicamente el contenido de los menús infantiles, en el contexto de una etapa de transición nutricional en la cual predomina el alto consumo de grasas saturadas, azúcares simples y baratos pero pobres en fibra. Considerando lo anterior, el objetivo fue, analizar el aporte calórico y de macronutrientes de los menús infantiles en restaurantes de comida rápida y restaurantes convencionales según tiempos de comidas en relación a requerimientos nutricionales para niños de 4 a 8 años en una ciudad del noreste de México la cual se ubica en una de las regiones mexicanas donde se ha registrado desde 1999 el mayor promedio de Índice de Masa Corporal $(\mathrm{IMC}=\mathrm{Kg} / \mathrm{m} 2)$ y con una prevalencia de obesidad infantil de $28,6 \%$ (las niñas $31,3 \%$ y los niños $25,5 \%$ ) (38).

\section{MATERIAL Y MÉTODOS}

Se trata de un estudio observacional, transversal y analítico; las unidades de observación fueron los menús infantiles que ofertan restaurantes que trabajan bajo el Sistema de servicio rápido y menús infantiles ofrecidos en restaurantes convencionales. Se realizó en el periodo de diciembre 2009 y febrero 2010 en el área metropolitana de Monterrey (AMM) la cual incluye 9 municipios: Apodaca, García, San Pedro Garza García, General Escobedo, Guadalupe, Juárez, Monterrey, San Nicolás de los Garza y Santa Catarina. Se incluyeron las cadenas de restaurantes de mayor presencia (mayor número de establecimientos) y establecidos en el AMM. Se tomaron como criterios de inclusión, los menús infantiles ofertados en establecimientos de comida rápida transnacional (franquicias extranjeras) y los menús infantiles de restaurantes convencionales que pertenecieran a cadenas reconocidas (franquicias nacionales).

Por conveniencia se seleccionaron 4 cadenas de comida rápida (117 restaurantes) y 4 cadenas de comida convencional (35 restaurantes). Se estudiaron la totalidad de los combos alimenticios (paquetes que incluyen por lo general un platillo y una bebida) ofrecidos en los menús infantiles de las cuatro cadenas de comida rápida (CR): CR no.1: 3 combos; CR no. 2: 3 combos; CR no.3: 4 combos; CR no. 4: 2 combos. También, se estudiaron 39 combos alimenticios de las cuatro cadenas de restaurantes convencionales (CC) los cuales constituían la totalidad de combos ofertados en el menú infantil en esos establecimientos: CC no.1: 7 combos; CC no. 2: 6 combos; CC no.3: 9 combos y CC no.4: 17 combos. Para determinar el valor nutritivo de los alimentos se utilizó el Laboratorio de Análisis de Alimentos, de la Facultad de Salud Pública y Nutrición UANL. El peso de los alimentos se determinó con una báscula Sartorius BL1500; la medición de los líquidos se hizo con un vaso de precipitado. Se llevó a cabo el siguiente procedimiento: 1) los combos alimenticios se llevaron al laboratorio inmediatamente después de su compra; 2) se separó cada uno de los componentes del combo y de los platillos; 3) los componentes sólidos eran pesados en gramos y registrados; 4) los alimentos líquidos eran medidos en mililitros y registrados; 5) se estableció el peso neto y el peso bruto de cada alimento registrado; 6) se codificó el alimento en gramos o mililitros de acuerdo al Sistema Mexicano de equivalentes, tomando en cuenta que en el sistema NUTRIS todos los alimentos están en crudo, 7) se evaluó la composición de los siguientes nutrimentos: energía (Kcal), carbohidratos (g), proteínas $(\mathrm{g})$ y grasa $(\mathrm{g})$; mediante el programa NUTRIS@® Versión 2.0 Sistema de Evaluación Dietética y Antropométrica (Versión para Windows).

Se tomaron como referencia valores e Ingesta Recomendada (IR) establecidos por el Food and Nutrition Board (FNB) Institute of Medicine (IOM) of The National Academies y validados para población mexicana por el Instituto Nacional de Ciencias Médicas y Nutrición Salvador Zubirán (INNSZ) (39). Los indicadores que se analizaron en los menús infantiles fueron: el aporte de las kilocalorías de acuerdo al requerimiento diario de 1,800 calorías para niños de 4 a 8 años, el aporte de los hidratos de carbono de acuerdo a la IR de 130 g/día, proteína de acuerdo a la IR de $19 \mathrm{~g} /$ día y, las grasas de acuerdo a la IR de $25 \mathrm{~g} /$ día. Asimismo, se analizó el aporte de las kilocalorías de los menús infantiles según el tiempo de comida en el cual fueran consumidos y de acuerdo a la siguiente distribución recomendada $(40,41): 25 \%$ desayuno, $30 \%$ comida, $15 \%$ colación y $30 \%$ cena.

Para llevar a cabo el análisis estadístico de los datos, los restaurantes se estratificaron en dos tipos: los de comida rápida y los de comida convencional. Los platillos se agruparon de acuerdo a cada una de ellas calculando el promedio y desviación estándar de macronutrientes que aportaron los alimentos de los platillos; los promedios obtenidos por cadena expendedora, se evaluaron de acuerdo a la ingesta recomendada (IR).

\section{RESULTADOS}

Los menús infantiles ofrecidos en los restaurantes de comida rápida aportaron entre el 29,3\% (CC no.4) y el 56\% (CR no.2) de la IR de las kilocalorías diarias de un niño de entre 4 y 8 años; los restaurantes convencionales aportaron entre el $29,3 \%$ (CC no.4) y el 40,7\% (CC no.3) (tabla 1).

Con respecto a los aportes de los hidratos de carbono (tabla 2), estos variaron en los restaurantes de comida rápida del $60,7 \%$ al $97,9 \%$ de la IR, mientras que, en los restaurantes de comida convencional del 53,8\% al 68,4\%.

En cuanto a la cantidad de proteínas (tabla 3), los menús de los restaurantes de comida rápida aportaron de $82,2 \%$ a $173 \%$ de la IR; en los restaurantes de comida convencional el aporte fue de $89,1 \%$ al $153 \%$ de la IR.

Con respecto a los lípidos (tabla 5), el análisis dietético, mostró que todos los restaurantes de comida rápida rebasan 
el $100 \%$ pues aportaron desde un $107,5 \%$ y hasta $165,3 \%$ de la IR. En los restaurantes de comida convencional, el aporte fue del $63 \%$ al $108,4 \%$.

Otra parte importante de los resultados de esta investigación lo constituye el análisis sobre el aporte de calorías de los menús seleccionados de acuerdo al porcentaje recomendado por tiempos de comida (tabla 5). En casi la totalidad de los tiempos de comida (desayuno, comida, colación y cena) el aporte calórico era superior al $100 \%$ de la IR, con rango de 97,8 \% (CC no.4 tiempo de comida y cena) y hasta $373 \%$ de la IR (CR no.2 en tiempo de colación).

\section{DISCUSIÓN}

El panorama general apunta a que los menús son hipercalóricos en ambos grupos, sin embargo, el promedio energético de los restaurantes de comida rápida (CR) es más elevado que el de los de comida convencional (CC); los casos de menor exceso en el aporte calórico (de acuerdo a la distribución porcentual para 4 comidas diarias) se ubican entre los de CC.

Los aportes de los hidratos de carbono fueron similares para ambos tipos de restaurantes. Se observó que los menús contienen una cantidad excesiva de hidratos de carbono (HC), los que además son en su mayoría simples; esto aplica a todos los restaurantes. Se aprecia que tan sólo en una comida los niños pueden llegar a consumir desde poco más de la mitad y hasta casi el $100 \%$ de la IR de HC. Estos valores son más elevados que los encontrados en estudios que han analizado el consumo total de energía entre niños que consumen habitualmente comida rápida (19). Existe además una tendencia hiperproteica en ambos grupos analizados y siendo más marcada en los combos de comida rápida.

Por otro lado, los combos son muy altos en lípidos, sin embargo, las comidas rápidas en su totalidad sobrepasan el $100 \%$ de la IR mientras que para los convencionales sólo dos lo sobrepasaron. Es necesario señalar que el exceso de lípidos se presenta combinado con un exceso de proteína animal y de hidratos de carbono, lo cual se ha asociado con el aumento de riesgo de obesidad, diabetes mellitus y enfermedades coronarias $(17,18,42)$. Se debe considerar que también podría haber un impacto en el establecimiento de hábitos alimentarios de los niños y que podrían verse reflejados en el gusto y/o preferencia alimentarios en la vida adulta $(17,42)$.

\section{TABLA 1}

Calorías según tipo de restaurante en una ciudad del noreste de México.

\begin{tabular}{lccc}
\hline Tipo de restaurante ${ }^{1}$ & $N^{\circ}$ de platillos & Aporte calórico Kcal/día ${ }^{2}$ & $\begin{array}{c}\text { Porcentaje del } \\
\text { aporte según la IR }\end{array}$ \\
CR No.1 & 3 & $714,4 \pm 244,0$ & 39,7 \\
CR No.2 & 3 & $1008,2 \pm 284,1$ & 56,0 \\
CR No.3 & 4 & $831,7 \pm 303,1$ & 46,2 \\
CR No.4 & 2 & $632,4 \pm 261,8$ & 35,1 \\
CC No.1 & 7 & $560,2 \pm 182,8$ & 31,1 \\
CC No.2 & 6 & $690,5 \pm 387,2$ & 38,4 \\
CC No.3 & 9 & $731,8 \pm 333,0$ & 40,7 \\
CC No.4 & 17 & $528,3 \pm 189,1$ & 29,3 \\
\hline
\end{tabular}

1. $C R=$ Comida rápida; $C C=C o m i d a$ convencional; 2 .Promedio $\pm 1 D S ; 3$. IR=Ingesta recomendada de 1,800 Kilocalorías/día, según Food and Nutrition Board, Institute of Medicine, National Academies, Food and Nutrition Center (39).

\section{TABLA 2}

Hidratos de carbono según tipo de restaurante en una ciudad del noreste de México.

\begin{tabular}{cccc}
\hline Tipo de restaurante & N $^{1}$ de platillos & $\begin{array}{c}\text { Aporte de hidratos } \\
\text { de carbono (g/día) }\end{array}$ & $\begin{array}{c}\text { Porcentaje del aporte } \\
\text { según la IR }\end{array}$ \\
CR No.1 & 3 & $97,7 \pm 48,3$ & 75,2 \\
CR No.2 & 3 & $127,3 \pm 35,8$ & 97,9 \\
CR No.3 & 4 & $92,6 \pm 31,9$ & 71,3 \\
CR No.4 & 2 & $78,9 \pm 28,1$ & 60,7 \\
CC No.1 & 7 & $88,9 \pm 29,1$ & 68,4 \\
CC No.2 & 6 & $82,8 \pm 47,4$ & 63,7 \\
CC No.3 & 9 & $69,9 \pm 47,9$ & 53,8 \\
CC No.4 & 17 & $71,5 \pm 26,2$ & 55
\end{tabular}

1. $C R=$ Comida rápida; $C C=$ Comida convencional; 2 . Promedio $\pm 1 D S ; 3$. IR=Ingesta recomendada de 130g/día según Food and Nutrition Board,

Institute of Medicine, National Academies, Food and Nutrition Center (39). 
Un aspecto relevante a considerar del consumo excesivo de calorías en menores de edad, es que en México existe un desconocimiento sobre los hábitos alimentarios de niños y niñas y en particular sobre estadísticas desagregadas sobre el hábito del desayuno en casa. Se debe tomar en cuenta que los restaurantes de comida rápida ofrecen un horario de servicio muy amplio, de 7 am a 10 pm, y que aproximadamente un $40 \%$ de los establecimientos dan servicio las 24 horas del día todos los días del año, lo cual permite que desde muy temprano y hasta altas horas de la noche se pueda tener acceso a comida hipercalórica. Lo anterior impone un cuestionamiento respecto a en qué medida los horarios de servicio de este tipo de establecimientos favorecerían la adquisición de los desayunos y de los otros tiempos de comida, en horarios no apropiados desde el punto de vista fisiológico y constituirían un factor de riesgo de obesidad.

Es posible que la ingesta de esos menús infantiles puedan tener un impacto en el estado nutricional dependiendo del tiempo de comida en que se consuman, dado que, se describen porcentajes en la distribución de la ingesta diaria de los alimentos $y$, por ende, de las calorías que estos aportan (32).

Se recomienda que el desayuno debe aportar $25 \%$ de las calorías totales, esto es, una ingesta de 450 calorías. Al analizar los aportes de las calorías que tienen los menús, se observó que para este tiempo de comida, los menús de las comidas convencionales pueden aportar desde un $117,4 \%$ y hasta un $162,6 \%$ en exceso, mientras que en los menús de las comidas rápidas pueden aportar de un 140,5\% hasta un $224 \%$ en exceso. Se debe tomar en cuenta que el exceso de calorías consumidas podrían ser utilizadas además de las funciones fisiológicas en actividades propias de los niños, de lo contrario, significa que ese exceso podría aumentar el tejido adiposo (32).

Así, para el tiempo de "colación" se recomienda el 15\% del total de las calorías, esto es 270 calorías. Si el consumo de los menús infantiles sucede durante el tiempo de la "colación", los porcentajes de exceso aumentan considerablemente. Los menús de las comidas convencionales para este tiempo de comida (colación) pueden ir del 195,7\% al 271\% en exceso, mientras que, los menús de las comidas rápidas, pueden aportar desde un $234,2 \%$ y hasta un $373,4 \%$ en exceso. En caso de que la colación fuese por la mañana, hay que recordar, que probablemente el niño ya ha desayunado y, si la colación fuera a media tarde (lo cual seguramente el niño ejerció los tiempos del desayuno y de la comida), se puede considerar que tiene

\section{TABLA 3}

Proteína según tipo de restaurante en una ciudad del noreste de México.

\begin{tabular}{cccc}
\hline Tipo de restaurante ${ }^{1}$ & $N^{0}$ de platillos & Aporte de proteína $(\mathrm{g} / \mathrm{día})^{2}$ & $\begin{array}{c}\text { Porcentaje del aporte } \\
\text { según la IR }\end{array}$ \\
CR No.1 & 3 & $15,6 \pm 1,1$ & 82,2 \\
CR No.2 & 3 & $32,9 \pm 14,2$ & 173,0 \\
CR No.3 & 4 & $25,0 \pm 10,4$ & 131,8 \\
CR No.4 & 2 & $18,9 \pm 12,2$ & 99,6 \\
CC No.1 & 7 & $16,9 \pm 5,7$ & 89,1 \\
CC No.2 & 6 & $29,2 \pm 16,7$ & 153,9 \\
CC No.3 & 9 & $23,1 \pm 18,0$ & 121,4 \\
CC No.4 & 17 & $20,0 \pm 10,3$ & 105,2
\end{tabular}

1. $C R=$ Comida rápida; $C C=$ Comida convencional; 2. Promedio $\pm 1 D S ; 3$. IR=Ingesta recomendada de 19g/día según Food and Nutrition Board, Institute of Medicine, National Academies, Food and Nutrition Center (39).

TABLA 4

Lípidos según tipo de restaurante en una ciudad del noreste de México.

\begin{tabular}{|c|c|c|c|}
\hline Tipo de restaurante ${ }^{1}$ & $\mathrm{~N}^{\circ}$ de platillos & Aporte de lípidos (g/día)² & $\begin{array}{c}\text { Porcentaje del aporte } \\
\text { según la } I \mathbb{R}^{3}\end{array}$ \\
\hline CR No.1 & 3 & $28,0 \pm 5,0$ & 112,0 \\
\hline CR No.2 & 3 & $41,3 \pm 11,1$ & 165,3 \\
\hline CR No.3 & 4 & $40,1 \pm 16,0$ & 160,5 \\
\hline CR No.4 & 2 & $26,9 \pm 12,4$ & 107,5 \\
\hline CC No.1 & 7 & $15,8 \pm 13,5$ & 63,0 \\
\hline CC No.2 & 6 & $26,6 \pm 17,3$ & 106,5 \\
\hline CC No.3 & 9 & $27,1 \pm 23,9$ & 108,4 \\
\hline CC No.4 & 17 & $16,7 \pm 9,9$ & 66,7 \\
\hline
\end{tabular}

1. $C R=C o m i d a$ rápida; $C C=C o m i d a$ convencional; 2 . Promedio $\pm 1 D S ; 3$. IR=Ingesta recomendada de $25 \mathrm{~g} /$ día según Food and Nutrition Board, Institute of Medicine, National Academies, Food and Nutrition Center (39). 
mayor efecto ya que se reduce el tiempo para poder utilizar o gastar el exceso de las calorías consumidas.

Al tiempo de la "comida" se recomienda el 30\% del total de las calorías, esto es un aporte de 540 calorías, el consumo de los menús infantiles en este tiempo, los excesos en las calorías pueden ir del $97,8 \%$ al $103.7 \%$ en los menús de las comidas convencionales $y$, en los menús de las comidas rápidas, el exceso de las calorías entre $117,1 \%$ y $186,7 \%$.

En estas condiciones, si los tiempos de comida que anteceden a la colación cumplen con los requerimientos, esto es, que no rebasan lo recomendado, los niños habrían consumido hasta ese momento el 55\% del requerimiento diario, o bien, si no tomó el desayuno, habría cubierto el 30\% del requerimiento calórico del día.

Al tiempo de la "cena", también le corresponde el 30\% del requerimiento diario, a los porcentajes de ambos tipos de menús les corresponderían los mismos excesos señalados más arriba en el tiempo de comida. Sin embargo, las condiciones respecto al consumo de las calorías podrían ser muy diferentes a los otros tiempos de comida, ya que, en este momento los niños ya habrían consumido, si se distribuye según lo recomendado, el 70\% del requerimiento de las calorías diarias. Debido a que posterior a este tiempo de comida (cena), las probabilidades de realizar algún tipo de actividad son relativamente bajas, el exceso de alimentos sería almacenado y si este patrón fuera constante podría haber implicaciones relacionadas con el exceso de peso.

Los resultados de este estudio tienen importantes implicaciones para los restaurantes, los padres de familia, los niños y los profesionales de la salud, pues se demuestra que, tanto los restaurantes de comida rápida como los restaurantes convencionales ofrecen menús infantiles hipercalóricos en un contexto donde cada vez más familias comen fuera de casa, lo que ilustra bien una de las manifestaciones más claras de la transición nutricional en esta época tal y como lo ha explicado Popkin (15).

Los resultados contrastan en un aspecto con el estudio de Serrano y Jedda (43), pues aunque encontraron similitud en cuanto al contenido calórico de menús infantiles de comida rápida y convencional, concluyeron que los restaurantes de comida rápida ofrecen a los niños porciones más pequeñas y con menor contenido de grasa que los restaurantes convencionales.
Existen coincidencias con diversos estudios (29-36) que asocian comida rápida y malnutrición por exceso. Pone de manifiesto lo engañoso que puede ser creer que sólo los restaurantes de comida rápida ofrecen alimentos y porciones poco saludables y abre la interrogante de por qué, en general, los restaurantes no han diseñado opciones de menú saludables para niños, tal y como algunos ya han hecho para el menú dirigido a los adultos ofertándoles platillos bajos en grasa, sal e hidratos de carbono y con información nutrimental detallada. Así, los resultados obtenidos apoyan la afirmación de que la industria de restoranes juega un papel fundamental en materia salud (44).

Es importante señalar que nuestro estudio, al igual que la mayoría de las investigaciones realizadas sobre comida rápida, se ha enfocado al análisis del aporte macronutrimental, por lo cual sería pertinente que en el futuro se lleven a cabo estudios longitudinales que tomen en cuenta el aporte de micronutrientes, así como también considerar otras variables como la distribución espacial de los diferentes tipos de restaurantes y el grado de marginación o estrato socioeconómico de los consumidores.

\section{RESUMEN}

Se analizó el aporte calórico y de macronutrientes de menús infantiles en restaurantes de comida rápida y restaurantes convencionales según tiempos de comidas e ingesta recomendada para niños de 4 a 8 años en una ciudad del noreste mexicano que presenta una prevalencia de obesidad infantil de las más altas del mundo. Es un estudio observacional, descriptivo y transversal. El muestreo fue por conveniencia y dirigido al ser seleccionados casos típicos de menús infantiles de cadenas de restaurantes de mayor presencia (152 establecimientos). Los resultados muestran que el rango de aporte de hidratos de carbono varió del 53,8\% a 97,9\% de la recomendación; el aporte de proteína no fue menor al 82,2\%; el aporte de lípidos fue de $63.0 \%$ a $165,3 \%$. Estos aportes aumentan considerablemente al analizarlos por tiempos de comida: de $97,8 \%$ a $373,4 \%$. Se concluye que tanto los restaurantes de comida rápida como los restaurantes convencionales ofrecen a la población infantil alimentos y porciones poco saludables.

Palabras clave: aporte calórico y macronutrimental; comida convencional; comida rápida; menús infantiles; nutrición infantil.

\section{TABLA 5}

Porcentaje de adecuación de calorías en los diferentes tiempos de comida según tipo de restaurante en una ciudad del noreste de México.

\begin{tabular}{|c|c|c|c|c|c|}
\hline Tipo de restaurante ${ }^{1}$ & $\mathrm{~N}^{\circ}$ de platillos & Desayuno 2 & Comida $^{3}$ & Colación ${ }^{4}$ & Cena $^{3}$ \\
\hline CR No.1 & 3 & 158,8 & 132,3 & 264,6 & 132,3 \\
\hline CR No.2 & 3 & 224,0 & 186,7 & 373,4 & 186,7 \\
\hline CR No.3 & 4 & 184,8 & 154,0 & 308,0 & 154,0 \\
\hline CR No.4 & 2 & 140,5 & 117,1 & 234,2 & 117,1 \\
\hline CC No.1 & 7 & 124,5 & 103,7 & 207,5 & 103,7 \\
\hline CC No.2 & 6 & 153,4 & 127,9 & 255,7 & 127,9 \\
\hline CC No.3 & 9 & 162,6 & 135,5 & 271,0 & 135,5 \\
\hline CC No.4 & 17 & 117,4 & 97,8 & 195,7 & 97,8 \\
\hline
\end{tabular}

1. $C R=$ Comida rápida; $C C=$ Comida convencional; 2 . Corresponde al $25 \%$ de la Ingesta Recomendada (IR);

3. Corresponde al $30 \%$ de la IR; 4 . Corresponde al $15 \%$ de la IR. 


\section{BIBLIOGRAFÍA}

1. Instituto Nacional de Estadística, Geografía e Informática. Encuesta Nacional de Ingresos y Gastos de los Hogares (ENIGH) 2002. México: INEGI; 2003.

2. Instituto Nacional de Estadística, Geografía e Informática. Encuesta Nacional de Ingresos y Gastos de los Hogares (ENIGH) 2004. México: INEGI; 2005.

3. Instituto Nacional de Estadística, Geografía e Informática. Encuesta Nacional de Ingresos y Gastos de los Hogares (ENIGH) 2006. México: INEGI; 2007.

4. Instituto Nacional de Estadística, Geografía e Informática. Encuesta Nacional de Ingresos y Gastos de los Hogares (ENIGH) 2008. México: INEGI; 2009.

5. Instituto Nacional de Estadística, Geografía e Informática. Encuesta Nacional de Ingresos y Gastos de los Hogares (ENIGH) 2010. México: INEGI; 2011.

6. Instituto Nacional de Estadística, Geografía e Informática. VIII Censo de Servicios 1981. Aguascalientes, México: INEGI; 1988.

7. Instituto Nacional de Estadística, Geografía e Informática. IX Censo de Servicios 1986. Censo Económico 1986. Aguascalientes, México: INEGI; 1991.

8. Instituto Nacional de Estadística, Geografía e Informática. X Censo de Servicios 1989, Censo Económico 1989. Aguascalientes, México: INEGI; 1993.

9. Instituto Nacional de Estadística, Geografía e Informática. XI Censo de Servicios 1994. Censo Económico 1994. México: INEGI; 1996.

10. Instituto Nacional de Estadística, Geografía e Informática. XII Censo de Servicios 1999. Censo Económico 1999. México: INEGI; 2002.

11. Instituto Nacional de Estadística, Geografía e Informática. XIII Censo de Servicios 2004.Censo Económico 2004. México: INEGI; 2006.

12. Instituto Nacional de Estadística, Geografía e Informática. Censos Económicos 2009. México: INEGI; 2010.

13. Instituto Nacional de Estadística, Geografía e Informática. Sistema de Clasificación Industrial para América del Norte, México. SCIAN 2007. México: INEGI; 2007.

14. Barrado EE. Composición grasa de diversos alimentos servidos en establecimientos de "comida rápida". Nutr Hosp 2008; 23(2):148-58.

15. Popkin M. B. Nutritional Patterns and Transitions. PDR 1993; 19 (1): 138-57.

16. Observatorio de Corporaciones Transnacionales (OCT). Boletín 6: El Sector de la Comida Rápida [monografía electrónica]. Disponible en: http://www.ideas.cooph descargas/cat_view/40-investigacion/43-boletinespbservatorio.html?start=10 Consultado el 05 de agosto de 2009.

17. Bauer LR., Waldrop J. Trans fat intake in children: risks and recommendations. Pediatr Nurs 2009; 35 (6): 346-52.

18. Lora K, Morse K, Gonzalez-Kruger GE, Driskell JA. High saturated fat and cholesterol intakes and abnormal plasma lipid concentrations observed in a group of 4-8 year old children of Latino immigrants in rural Nebraska. J Nut Res 2007; 27(8):483-91.

19. Bowman SA, Gortmaker $S L$, Ebbeling $C B$, Pereira $M A$, Ludwig DS. Effects of fast-food consumption on energy intake and diet quality among children in a National Household Survey. J Pediatr 2004; 113(1): 112-8.

20. Schlosser E. Fast Food Nation. Barcelona: Ramdom House Mondadori; 2003.

21. Story $M$, French S. Food advertising and marketing di- rected at children and adolescents in the US. Int J Behav Nutr Phys 2004; 1:3-20.

22. Page RM. Emotional and rational product appeals in televised food advertisements for children: analysis of commercial shown on US broadcast networks. J Child Health Care 2007; 11(4): 323-40.

23. Robinson TN, Borzekowski DL, Matheson DM, Kraemer HC. Effects of fast food branding on Young children's taste preferences. Arch Pediatr Adolesc Med 2007; 161(8): 792-7.

24. Chernin A. The effects of food marketing on children's preferences: testing the moderating roles of age and gender. ANNALS AAPSS 2008; 615(1): 101-18.

25. Linn S, Novosat CL. Calories for sale: food marketing to children en the twenty-first century. ANNALS, AAPSS 2008; 615(1): 133-5.

26. Dorey E., McCool J. The role of the media in influencing children's nutritional perceptions. Qual Health Res 2009; 19(5):645-54.

27. Roberto CA, Baik J, Harris JL, Brownell KD. Influence of licensed characters on children's taste and snack preferences. J Pediatr 2010; 126(1): 88-93.

28. Zimmerman FJ, Bell JF. Associations of television content type and obesity in children. Am J Public Health 2010; 100(2): 334-40.

29. Annuar Zaini MZ, Lim CT, Low WY, Harun F. Factors affecting nutritional status of Malaysian school children. Asia Pac J Public Health 2005; 17(2):71-80.

30. Kelly $L E$, Patterson BJ. Childhood nutrition: perceptions of caretakers in a low-income urban setting. J Sch Nurs 2006; 22(6): 345-51.

31. Wiecha J, Finkelstein D, Troped P, Fragala M, Peterson KE. School vending machine use and fast-food restaurant use are associated with sugar-sweetened beverage intake in youth. J Am Diet Assoc 2006; 106(10): 1624-30.

32. Tojo R, Leis, R. Alimentación del niño escolar [libro electrónico]. En: Suárez L, editora. Manual Práctico de nutrición en pediatría. Madrid: Editorial Ergon; 2007. Disponible en: www.nutrinfo.com.mx.

33. Freeman A. Fast food: oppression through poor nutrition. California Law Review 2007; 96(6): 2221-59.

34. Davis B, Azuza C. Fast food near schools means fatter kids. Ro J Med Pract 2009; 4(1):40-4.

35. Denney-Wilson E, Crawford D, Dobbins T, Hardy L, Okely A. Influences on consumption of soft drinks and fast food in adolescents. Asia Pac J Clin Nutr 2009; 18(3): 447-52.

36. Wood M. Kids, fast food and obesity. Agricultural Research 2009; 57(9): 20-21.

37. Rivera J, Shamah T, Villalpando S, González T, Hernández B, Sepúlveda J. Encuesta Nacional de Nutrición 1999. Estado nutricio de niños y mujeres en México. Cuernavaca, Morelos, México: Instituto Nacional de Salud Pública; 2001.

38. Olaiz G, Rivera J, Shamah $T$, Rojas $R$, Villalpando $S$, Hernández M, Sepúlveda J. Encuesta Nacional de Saludy Nutrición 2006. Cuernavaca, México: Instituto Nacional de Salud Pública; 2006.

39. Food and Nutrition Information Center (FNIC), United States Department of Agriculture, National Agricultural Library [homepage on the internet]. Dietary References Intakes: macronutrients. Disponible en: www.iom.edu Global/News\%20Announcements/ /media/C5CD2DD7840544979A549EC47E56A02B.ashx Consultado el 10 de agosto de 2009. 
40. Casanueva E. Nutriología Médica. México DF: Editorial Panamericana; 2001.

41. Escott-Stump S. Nutrición, diagnóstico y tratamiento. México DF: McGraw Hill; 2005.

42. Ricketts CD. Fat preferences, dietary fat intake and body composition in children. Eur J Clin Nutr 1997; 51:778-81.
43. Serrano $E$, Jedda V. Comparison of fast-food and non fast-food children's menu items. J Nut Educ Behav 2009; 41(2): 132-7.

44. Demory-Luce D. Fast food and children and adolescents: implications for practitioners. Clin Pediatr 2005; 44:279-88. 